OCD symptoms being solely concerned with COVID-related contamination. The questionnaires routinely completed at the time of assessment and treatment were the Obsessive Compulsive Inventory (OCI); Yale Brown Obsessive Compulsive Scale (YBOCS); Beck Depression Inventory (BDI). Clinical data were collated and analysed prior to and during the pandemic. Treatment consisted of ERP and was adapted for provision via a virtual platform. ERP involved exposure to a graded hierarchy of COVID-specific anxiety-provoking situations modified to take government guidelines into consideration.

Discussion. Prior to the COVID-19 pandemic the patient's response to treatment with cognitive behavioural therapy (CBT) including ERP indicated a $79 \%$ improvement in OCD symptoms on self -rated measures. The impact of the pandemic led to a significant $65 \%$ deterioration in OCD symptoms, regarding COVID-19 contamination concerns. Intervention with ERP resulted in $73 \%$ improvement over a three-month period. Measures of depression symptoms indicated an $80 \%$ improvement pre-COVID, with a $78 \%$ deterioration at relapse. Following treatment, the patient also showed a $65 \%$ improvement in depression symptoms. Improvements have been maintained at one month follow-up.

Conclusion. The case study supports literature indicating the exacerbation of OCD symptoms due to the COVID-19 pandemic for patients with contamination fears and washing compulsions. The promising results support the use of ERP as an effective treatment for COVID-related OCD symptoms. It also validates the provision of CBT interventions virtually to ensure accessibility of treatment to OCD sufferers.

\section{A brief novel intervention for acrophobia (fear of heights)}

\section{E. Naomi Smith*}

South London and Maudsley Training Scheme

${ }^{*}$ Corresponding author.

doi: 10.1192/bjo.2021.351

Objective. To investigate a unique brief intervention, which offers a combination of neuro-linguistic programming and practical graded exposure therapy, to overcome a fear of heights.

Background. A fear of heights or acrophobia is common and often deters people from perusing activities like climbing. It can also interfere with routine activities of daily living.

Case report. This two-day intervention is set in the Peak District (Derbyshire, UK) and works with a maximum of eight individuals to four instructors. The first half-day involves working with a psychotherapist using neurolinguistic programing techniques. The next 1.5 days involves graded exposure using abseiling over gradually increasing heights, to a final height of approximately 40 feet. Discussion. All eight individuals on the two-day course felt their fear of highs had significantly decreased. All eight individuals would recommend this intervention to others suffering from a fear of heights.

Conclusion. It is noteworthy that the group undergoing this intervention were self-selected and highly motivated to overcome their fear of heights. The sample size was small and outcome measures were subjective. However, this is a novel and effective approach to helping people overcome their fear of heights. Further research with larger sample sizes would be beneficial in further assessing the impact of this intervention.

Declaration: Permission was granted by the organizers of this intervention to submit an abstract to conference. There are no conflicts of interests. This intervention is run by a private company 'Will4Adventure', I have no finical or other interests in this company. I privately funded my own place on this course.

\section{Access to firearms: essential factor for risk management in psychiatry}

Donnchadh Walsh ${ }^{1 *}$, Una Fallon ${ }^{2}$, Sonn Patel ${ }^{3}$ and Elizabeth Walsh ${ }^{3}$

${ }^{1}$ School of Medicine, University College Dublin; ${ }^{2}$ Galway Menal Health Service and ${ }^{3}$ University Hospital Galway

${ }^{\star}$ Corresponding author.

doi: 10.1192/bjo.2021.352

Objective. Increase awareness of the risk associated with access to firearms in clinical practice.

Case report. A 57-year-old man, with a 30 year history of schizophrenia, was reviewed routinely at home. His illness is predominantly characterised by chronic delusions of a grandiose nature. He believes he has been offered various senior employment positions and has acted on these beliefs by presenting at workplaces in business attire. He has no insight into his condition. At review, he described awakening a week earlier in a panic and seizing hold of his legally held shotgun. He planned to shoot out the window as he believed people were breaking in. His wife prevented him from doing so by taking the gun and hiding it. A few days later he found the gun and intended to frighten off potential pursuers by pretending to shoot birds. He was persuaded to surrender the gun and it was taken to the local Garda (Police). A short time later, he presented to Garda Headquarters, over an hour away, seeking the return of his gun. At review, he had limited insight into the potential seriousness of the situation. The team immediately liaised with Gardaí, a HCR- 20 risk assessment was completed and clozapine levels checked.

Discussion. We had not know that our patient owned a shotgun despite very regular contact with him. During a comprehensive psychiatric history we routinely ask about risk of harm to self and others, but rarely ask specifically about access to or ownership of guns. Working on a farm, rural living or having an interest in shooting sports may raise the issue. Suicide, security breaches and homicide are the main risks conferred by firearms in mental illness. Mental illness is not necessarily prohibitive to gun ownership. Applicants for gun certificates in the UK must disclose specific medical conditions, including a psychotic illness, and an automatic medical report is sought. In the Irish Republic it is the responsibility of the applicant to declare any specific physical or mental health condition. Although a medical report may be sought, it is not automatic in all cases. Lack of insight into psychotic illness may potentially influence self-declaration upon application for a certificate.

Conclusion. Awareness of a persons access to firearms should be part of our routine risk assessment.

\section{Michael Kohlhaas Syndrome - Taking the court to court}

Louisa Ward*

Worcestershire Health and Care NHS trust ${ }^{*}$ Corresponding author.

doi: 10.1192/bjo.2021.353

Objective. To explore Michael Kohlhaas syndrome and development of litigious paranoia. 
Case report. A 50-year-old Swiss woman spent a number of weeks travelling around the UK, reporting to police that her family in Switzerland were working with their police, doctors and the mafia to kill her. She was therefore running away for her own safety, and trying to seek legal help to investigate. She had been in close contact with a woman in London who claimed to be able to give her legal aid in exchange for payment. On admission to the ward, it was felt that the legal aid was actually feeding her persecutory delusions.

Discussion. We discovered that she had in fact been detained in Switzerland prior to coming to the UK, and the discharge report was obtained which diagnosed her with Michael Kohlhaas syndrome and Folie a deux.

Conclusion. This poster will further explore Michael Kohlhaas syndrome and litigious paranoia, with connection to this case.

\section{Education and Training}

Trainees' perspective on the best use of supervision-hour in psychiatry training - a qualitative study

Raja Adnan Ahmed*, Mohamed Bader and Mohamed Flensham

Aneurin Bevan University Health Board

${ }^{\star}$ Corresponding author.

doi: 10.1192/bjo.2021.354

Aims. This study aims to identify the techniques to improve the quality of the weekly one to one supervision for Psychiatry trainees.

Method. An open-ended online questionnaire was prepared using principles of critical incident technique and distributed among psychiatry trainees working in various deaneries within the UK. The participants were asked to describe an example of a good and a bad supervision experience they had encountered during their training. In addition, participants were also requested to make suggestions to improve the supervision experience. All qualitative data were analysed using the thematic analysis approach, to identify common themes.

Result. A total of 53 trainees working in various deaneries across England and Wales, responded to the questionnaire. The respondents were at a different level of training in psychiatry from CT1-ST6 level. The supervision hour was reported to be useful for clinical case discussions, reflection on difficult cases and situations, pastoral support and wider issues relating to personal and professional development. Trainees appreciated a holistic scope for supervision rather than a narrow discussion of management of cases.

Trainees reported that the supervision hour should be trainee-led and tailored according to their unique learning needs. Participants also saw supervision hour as a safe space where they can receive constructive criticism and feedback on their performance. At times, trust and genuineness were appreciated, as well as the use of an informal tone by the supervisor. An effective supervision leads to trainees feeling valued.

Conclusion. Trainees acknowledged that the supervision hour is an effective tool in psychiatry training. Trainees should get regular, protected and uninterrupted time with consultants for weekly supervisions. Both trainees and trainers need to develop a better understanding of how this supervision experience could be improved and tailored to the individual learning needs of the trainee.
The person behind the label: co-production as a tool in teaching about borderline personality disorder

Nyakomi Adwok ${ }^{\star}$ and Sharon Nightingale

Leeds and York Partnership Foundation Trust

${ }^{*}$ Corresponding author.

doi: 10.1192/bjo.2021.355

Aims. The overarching aim of the session was to address and reduce stigma around Borderline Personality Disorder among doctors. The three main objectives were:

To increase empathy and understanding around Borderline Personality Disorder by exposing junior doctors to service user perspectives outside a clinical setting;

To address knowledge gaps identified by junior doctors in a self-reported questionnaire disseminated prior to the teaching session;

To offer junior doctors a basic psychological framework to base their assessment and formulation of service users with personality disorders.

Background. 'Borderline Personality Disorder: The Person Behind the Label' was the title of the first co-produced teaching session in the Leeds and York Partnership Foundation Trust (LYPFT). Prior to the teaching session, an online questionnaire was sent out to trainees. The results highlighted three key issues:

Negative attitudes towards service users with personality disorders;

Poor subjective knowledge of the psychological models of personality disorders;

Perception among trainees that they do not receive adequate training to deal with the challenges service users with personality disorders present.

Method. A teaching session was co-produced by a team of two service users, a principal clinical psychologist within the Leeds Personality Disorder Network (PDN) and a core Psychiatry trainee. It was delivered in a 75 minute session to 40 attendees consisting of both trainee doctors and consultants.

Result. Feedback was collected immediately after the session through the use of anonymous feedback forms. The response to the training was overwhelmingly positive with all 28 respondents rating the session as $4 / 5$ or $5 / 5$ on a satisfaction scale ranging from 1 (poor) to excellent (5). Key themes from the feedback included appreciation for the service user perspective and teaching on psychological theory. The fourth question in the questionnaire: "How will this teaching impact your work?" produced the highest number of responses $(25 / 28)$ and provided evidence that the above listed objectives of the session were met.

Conclusion. Co-produced teaching has great potential to address negative attitudes around highly stigmatised conditions by bridging the gap that often exists between service users and mental health professionals.

Provision of training for accommodation providers in the London Borough of Hackney - results of a scoping exercise and educational session pilot

Kate Aldersey ${ }^{\star}$, Sheraz Ahmad and Sian Mason

East London NHS Foundation Trust

${ }^{\star}$ Corresponding author.

doi: 10.1192/bjo.2021.356

Aims. To understand the level of training given to staff in providers of accommodation in the London Borough of Hackney across mental and physical health. 